\title{
Diferencias según sexo en los factores asociados a hipertensión arterial en el Perú: Análisis de la Encuesta Demográfica y de Salud Familiar 2017 Gender differences in factors associated with hypertension in Peru: Analysis of the National Demographic and Health Survey 2017
}

\author{
Milagros Romero Giraldo ${ }^{1, a}$, Jane Avendaño-Olivares ${ }^{1, b}$ Rodrigo Vargas-Fernández ${ }^{1, c}$, Fernando M. Runzer-Colmenares ${ }^{1, d}$ \\ ${ }^{1}$ Universidad Científica del Sur. Lima, Perú. \\ ${ }^{a}$ Estudiante de Medicina, ORCID: 0000-0002-0704-6429 \\ ${ }^{a}$ Estudiante de Medicina, ORCID: 0000-0001-9691-3039 \\ a Estudiante de Medicina, ORCID: 0000-0002-3310-8689 \\ ${ }^{\mathrm{d}}$ Médico geriatra, magister en docencia universitaria. ORCID: 0000-0002-7717-8996
}

\section{Correspondencia:}

Milagros Natali Romero Giraldo mili.romerog18@gmail.com

Recibido: 13 de septiembre 2019 Aceptado: 23 de diciembre 2019 Publicación en línea: 31 de marzo 2020

Conflictos de interés: Los autores declaran no tener conflictos de interés.

Fuentes de financiamiento:

Autofinanciado

Contribuciones de autoría: MRG y JAO tuvieron la idea de investigación y diseñaron el estudio. RVF recopiló, procesó y analizó los datos.

Todos los autores participaron en la interpretación de los datos, redacción del manuscrito, revisión crítica y aprobaron la versión final.

Citar como: Romero M, AvendañoOlivares J, Vargas-Fernández $R$, Runzer-Colmenares F. Diferencias según sexo en los factores asociados a hipertensión arterial en el Perú: Análisis de la Encuesta Demográfica y de Salud Familiar 2017. An Fac med. 2020;81(1):33-9. DOI: https://doi.org/10.15381/anales. v81i1.16724
An Fac med. 2020;81(1):33-9/ DOI: https://doi.org/10.15381/anales.v81i1.16724

\section{Resumen}

Introducción. La hipertensión arterial (HTA) es una de principales causas de muerte a nivel mundial, donde el sexo masculino es el grupo mayormente afectado. Objetivo. Determinar las diferencias según sexo de Ios factores asociados a HTA en la población peruana mayor de 18 años. Métodos. Se realizó un análisis secundario de la Encuesta Nacional y Demográfica de Salud 2017. Se calcularon razones de prevalencia crudas (RP) y ajustadas (RPa) y sus intervalos de confianza al 95\% (IC 95\%) mediante modelos lineales generalizados de la familia Poisson entre la variable dependiente (HTA) e independientes. Resultados. Se encontró que la prevalencia de HTA fue de $17,8 \%$ en hombres y $11,4 \%$ en mujeres. La edad, tener sobrepeso y obesidad aumentaron la probabilidad de padecer HTA. Mientras que ser del resto de la costa, sierra y selva disminuyeron la probabilidad de HTA en ambos sexos. Conclusiones. Existe mayor prevalencia de HTA en el sexo masculino y factores como la edad, sobrepeso y obesidad aumentaron la probabilidad de padecer HTA.

Palabras clave: Hipertensión; Sexo; Encuesta de Salud; Perú (fuente: DeCS BIREME).

\section{Abstract}

Introduction. High blood pressure (HBP) is a leading cause of death worldwide, where the male sex is the most affected group. Objetives. Determine the gender differences of the factors associated with HBP in the Peruvian population over 18 years of age. Methods. A secondary analysis of the 2017 National Demographic and Health Survey was performed. Crude (RP) and adjusted (RPa) prevalence ratios and 95\% confidence intervals $(95 \% \mathrm{Cl})$ were calculated using generalized linear models of the Poisson family between the dependent variable (HBP) and independent. Results. The prevalence of HBP was found to be $17,8 \%$ in men and $11,4 \%$ in women. Age, being overweight and obese increased the likelihood of HBP. While being from the rest of coast, mountain range and jungle decreased the likelihood of HBP in both sexes. Conclusions. There is a higher prevalence of HBP in the male sex and factors such as age, overweight and obesity increased the likelihood of HBP.

Keywords: Hypertension; Sex; Health Survey; Peru (Source: MeSH NLM). 


\section{INTRODUCCIÓN}

La hipertensión arterial (HTA) es un problema de salud pública a nivel mundial por ser uno de los principales factores de riesgo para el desarrollo de enfermedades cardiovasculares (ECV) ${ }^{(1)}$. En 2017, las ECV generaron más de un millón y medio de muertes y 330 millones de DALYs (disability adjusted life years, por sus siglas en inglés) a nivel mundial (2). Dentro de las principales causas de muerte, la HTA como factor de riesgo se ubicó en el primer lugar ${ }^{(3)}$. Ello, obliga a las autoridades sanitarias a priorizar presupuestos nacionales en medidas recuperativas y preventivo-promocionales en las poblaciones más afectadas.

A nivel mundial, la prevalencia de HTA ha ido en aumento produciendo más de 10 millones de muertes y 218 millones de DALYs en $2017^{(3)}$. Majid Ezzati et al. estimaron un aumento de 594 millones de personas en 1975 a 1,13 mil millones con HTA en $2015^{(4)}$, lo que genera una gran preocupación en los países y sus sistemas sanitarios. Para un control adecuado a partir de la prevención, se debe conocer la definición operativa de la HTA, entendida como una presión arterial sistólica mayor y/o igual a $140 \mathrm{mmHg}$ y/o presión arterial diastólica mayor y/o igual a $90 \mathrm{mmHg}{ }^{(5)}$. Por lo tanto, los médicos y profesionales de salud en general deben estar en constante seguimiento de pacientes que cumplan con esta definición y hacer un diagnóstico precoz y tratamiento efectivo para evitar complicaciones tempranas y tardías.

La HTA presenta diferencias en relación al sexo; así, se evidenció que en hombres generó 3963 muertes por cada 100000 y 82915 DALYs, en comparación con las mujeres con 3872 muertes y 60 122 DALYs en $2015^{(6)}$. En América Latina, Rubinsteinn et al. evidenciaron que la prevalencia de HTA fue mayor en hombres que en mujeres, con $46,6 \%$ y $38,7 \%$, respectivamente ${ }^{(7)}$. En Perú, se encontró el mismo patrón donde los hombres tuvieron una mayor prevalencia de HTA $(16,6 \%)$ comparado con las mujeres $(10,7 \%)$ en $2017^{(8)}$. Por ello, conocer los factores que influyen en la prevalencia de HTA según el sexo, ayudaría a generar políticas orientadas a la disminución de la carga de enfermedad por esta patología.
Asimismo, existen factores psico-socio-culturales que según estudios previos tienen asociación con la HTA y se establecen de acuerdo a la modificación de los mismos. Así, factores modificables como el consumo de tabaco y alcohol estuvieron asociados con una mayor prevalencia de HTA en varones ${ }^{(9)}$. De igual modo, se observó que un bajo nivel de educación, índice de masa corporal (IMC) aumentado y residentes en zonas urbanas estaban asociados con HTA ${ }^{(10)}$. Por otro lado, un mayor nivel de riqueza y el menor consumo de frutas y verduras, fueron asociados de forma positiva a HTA independientemente del sexo por otros estudios ${ }^{(10,11)}$. En cuanto a factores no modificables, la probabilidad de desarrollar HTA aumenta con la edad en ambos sexos, afectando más a los hombres ${ }^{(10)}$. Estos estudios demostraron la diversidad de factores modificables y no modificables relacionados al desarrollo de HTA y la necesidad de construir estrategias sanitarias aplicables en la atención primaria de la salud para el cambio de estilos de vida como lo plantea la Organización Panamericana de la Salud en su informe Hearts ${ }^{(12)}$.

En Perú, la Encuesta Demográfica y de Salud Familiar (ENDES) 2017 brinda datos descriptivos sobre los factores anteriormente mencionados sin diferenciarlos entre el sexo femenino y masculino ${ }^{(13)}$. A pesar de ello, no existe evidencia sobre la diferencia de magnitud en la asociación de factores de riesgo para la HTA según sexo. Por ello, el objetivo de este estudio fue determinar las diferencias según sexo de los factores asociados a hipertensión arterial en la población peruana mayor de 18 años.

\section{MÉTODOS}

\section{Diseño del estudio}

Se realizó un estudio analítico de corte transversal a partir de los datos de 30682 personas, de las cuales 13466 eran hombres y 17216 mujeres mayores de 18 años, de la ENDES $2017^{(11)}$, siguiendo las recomendaciones contenidas en la guía SAGER (12). ENDES es una encuesta de base poblacional con representación nacional, departamental y por área urbano y rural, que recopila información sobre las enfermedades crónicas no transmisibles y el acceso a servicios diagnósticos y de tratamiento en el Perú. El tipo de muestreo es bietápico, probabilístico e independiente. Los detalles del muestreo, procesamiento y recolección de datos se encuentran en el informe técnico de la ENDES.

\section{Variables de estudio}

La variable dependiente fue la presencia de HTA definida como una presión sistólica $\geq 140 \mathrm{mmHg}$ y/o diastólica $\geq 90$ $\mathrm{mmHg}{ }^{(13)}$ y fue dicotomizada en 1 si tenía HTA, y 0 cuando no la tenía. Para considerar el valor de la presión arterial, se utilizó el promedio de dos tomas realizadas por el personal entrevistador previamente capacitado mediante el manual de la entrevistadora dado por el Instituto Nacional de Estadística e Informática (INEI), donde se detalla cómo se debe realizar una toma de presión arterial adecuada ${ }^{(14)}$

Por otro lado, las variables independientes de interés fueron edad, sexo, estado civil, área de residencia, nivel educativo, dominio de residencia, diabetes, quintil de bienestar, índice de masa corporal, consumo de alcohol y tabaco. La creación y selección de las variables se realizó teniendo en cuenta estudios previamente publicados y de interés epidemiológico ${ }^{(15,16,17)}$.

\section{Análisis estadístico}

Todos los análisis se realizaron mediante el programa estadístico Stata ${ }^{\circledR}$ 14.2 (Stata Corp, College Station, TX, USA). Para el análisis descriptivo, se usaron medias con su desviación estándar para las variables cuantitativas, y frecuencias absolutas y proporciones ponderadas para las variables categóricas. La asociación entre las variables de estudio se determinó mediante la prueba de chi-cuadrado. Se estimaron medidas de asociación mediante modelos lineales generalizados (MLG) de la familia Poisson con función de enlace (log) para reportar razones de prevalencia crudos (RP) y ajustados (RPa) y sus intervalos de confianza al 95\% (IC 95\%) entre la variable dependiente (presencia de HTA) e independientes. Para la inclusión de las variables independientes en el modelo ajustado debían tener un valor de $p<0,20$ en el 
análisis crudo. Finalmente, se consideró un valor de $\mathrm{p}<0,05$ como estadísticamente significativo.

\section{Aspectos éticos}

El presente estudio fue aprobado por el Comité de Ética de la Universidad Científica del Sur mediante el código de registro N² 286-2018-PRE2015.

\section{RESULTADOS}

Se incluyó un total de 30682 registros de personas en el análisis, con edad media de 40 años, predominó el sexo femenino $(51,1 \%)$, la mayoría eran casados o convivientes (68,3\%), del área urbana $(79,7 \%)$, cerca de la mitad tenían educación secundaria completa $(41,1 \%)$ y pertenecían al quintil 3 (21,1\%). Asimismo, el 40,8\% tenía sobrepeso, 37\% procedía de Lima, 14,5\% presentó hipertensión arterial, 36,1\% consumía alcohol, 11,6\% tabaco y sólo 3,5\% reportó tener antecedente de diabetes como comorbilidad (Tabla 1).

La edad media del sexo femenino fue de 40,2, mientras que del sexo masculino fue de 41,7 . El $72,1 \%$ de hombres y $64,7 \%$ de mujeres eran casados o convivientes, $80 \%$ y $79,4 \%$ de mujeres y hombres residían en el área urbana, respectivamente. En el nivel educativo, la educación secundaria predominó en ambos sexos. En cuanto al IMC, los hombres y mujeres tuvieron sobrepeso en mayor proporción. Las mujeres padecen diabetes en mayor porcentaje (3,9\%). En contraste con los varones que sufrieron HTA en mayor proporción $(17,8 \%)$ de los hombres que la padecieron. Los hombres consumían más tabaco $(19,3 \%)$ y alcohol $(46,9 \%)$ a diferencia de las mujeres $(4,1 \%$ y $25,7 \%$, respectivamente). Por último, ambos sexos presentaron proporciones similares en relación al quintil de bienestar (Tabla 1).

En la Tabla 2 se reporta el análisis crudo para los factores asociados a la HTA en mujeres. Se encontró asociación significativa con edad, nivel de educación, dominio de residencia, diabetes y consumo de alcohol, mientras que, área de residencia, quintil de bienestar, consumo de tabaco e IMC no tuvieron asociación significativa. En el modelo ajustado, se encontró que la edad ( $\mathrm{RPa}=1,05$; IC 95\%: 1,05-1,06), nivel de educación primaria ( $R P a=1,22 ;$ IC 95\%: 1,02-1,45), sobrepeso (RPa=1,86; IC 95\%: 1,15-3,01), obesidad (RPa=2,36; IC 95\%: 1,46-3,81) y diabetes (RPa=1,34; IC 95\%: 1,05-1,70) tuvieron mayor probabilidad de padecer HTA comparado a no tener educación, bajo peso y no presentar diabetes. Por otro lado, se encontró que ser del resto de la costa (RPa=0,74; IC 95\%: 0,61-0,89), sierra ( $R P a=0,58$; IC 95\%: 0,46-0,42), selva (RPa=0,79; IC 95\%: 0,63-0,99), pertenecer al quintil 2 (RPa=0,83 IC 95\%: $0,69-0,99)$ y quintil 3 (RPa=0,67; IC 95\%: 0,54-0,85) tuvieron mayor probabilidad de padecer HTA en comparación con ser de Lima Metropolitana y del quintil más pobre, respectivamente.

En el análisis crudo en varones (Tabla 3), se encontró asociación en todas las variables, excepto peso normal $(p=0,822)$, sobrepeso $(p=0,087)$, consumo de alcohol ( $p=0,369)$, quintil $2(p=0,526)$ y quintil $3(p=0,458)$. En el modelo ajustado, se encontró que la edad ( $R P a=1,03 ;$ IC 95\%: 1,03-1,03), estado civil (otro) ( $\mathrm{RPa}=1,41$; IC 95\%: 1,24-1,61), sobrepeso ( $\mathrm{RPa}=1,75$; IC 95\%: 1,01-3,02) y obesidad ( $\mathrm{RPa}=2,84$; IC 95\%: 1,64-4,91) presentaron mayor probabilidad de padecer HTA comparado al estado civil (casado/conviviente) y bajo peso. Por otro lado, se encontró que pertenecer al resto de la costa $(\mathrm{RPa}=0,80$; IC 95\%: 0,69-0,92), sierra ( $\mathrm{RPa}=0,73$; IC 95\%: 0,61-0,84), selva ( $R P a=0,77$; IC 95\%: 0,64-0,93) y quintil 3 (RPa=0,76 IC 95\%: 0,61-0,95) presentaron menor probabilidad de padecer HTA.

\section{DISCUSIÓN}

Nuestro estudio encontró mayor prevalencia de HTA en hombres con una diferencia de 6,4 puntos porcentuales, lo que concuerda con reportes similares a nivel mundial ${ }^{(18)}$. Asimismo, los factores asociados a HTA en ambos sexos fueron edad, nivel de educación primaria, sobrepeso, obesidad y dominio de residencia. Otros factores asociados a HTA fueron diabetes (solo en mujeres) y quintiles de bienestar (Q2 y Q3 en mujeres, Q3 y Q4 en varones).

Existen factores modificables y no modificables asociados a la HTA. En el es- tudio se evidenció que el sexo y la edad fueron los únicos factores no modificables abordados. Así, se evidenció que el sexo masculino tuvo mayor prevalencia que las mujeres, lo que puede explicarse en base a teorías genéticas e inmunológicas, donde se plantea que el estradiol endógeno en mujeres premenopáusicas posee un papel protector ${ }^{(19)}$. Asimismo, estudios en animales plantean que existen diferencias en cuanto al sexo en células $T$ y en la activación del sistema renina - angiotensina el cual puede contribuir a una mayor presión arterial en hombres ${ }^{(20)}$. En dicho estudio se propone que el perfil inmune anti inflamatorio en mujeres puede actuar como mecanismo compensatorio para limitar el aumento de presión en comparación de hombres quienes muestran mayor actividad proinflamatoria ${ }^{(20)}$.

Otro factor no modificable en el estudio fue la edad, donde la relación entre HTA y edad muestra una asociación positiva en ambos sexos, lo que concuerda con estudios previos, que plantean que el aumento de la presión arterial se hace evidenciable en la adolescencia persistiendo en la adultez, e incrementándose en la etapa adulta mayor ${ }^{(21)}$.

Por otro lado, dentro de los factores modificables y donde se aplican la mayoría de estrategias sanitarias, se encuentra el consumo de tabaco, que según nuestros resultados no fue significativo en ninguno de los géneros, tal vez por la baja prevalencia de tabaquismo en la población peruana ${ }^{(22)}$; sin embargo, estudios anteriores lo han documentado como un factor importante para desarrollar HTA tanto en varones como mujeres ${ }^{(23)}$.

De igual modo, se encontró asociación positiva entre IMC e HTA, siendo el sobrepeso y la obesidad, los factores de mayor prevalencia. Francesco Landi, et al. evidenciaron resultados similares a nuestro estudio con una mayor asociación entre las personas con sobrepeso y obesidad que la población general (24). Por otro lado, el IMC constituye un estimador de riesgo de enfermedad cardiovascular, particularmente por su asociación con la HTA. Por tal motivo, es importante hacer énfasis en la implementación de medidas de promoción y prevención en salud en 
Tabla 1. Características sociodemográficas y prevalencia de HTA en adultos peruanos, ENDES 2017.

\begin{tabular}{|c|c|c|c|c|c|c|c|}
\hline \multirow[b]{2}{*}{ Características } & \multicolumn{2}{|c|}{ Población adulta } & \multicolumn{2}{|c|}{ Mujeres } & \multicolumn{2}{|c|}{ Hombres } & \multirow[b]{2}{*}{ Valor de $p$} \\
\hline & $\mathbf{n}$ & $\begin{array}{l}\text { Proporción } \\
\text { ponderada }\end{array}$ & $\mathbf{n}$ & $\begin{array}{l}\text { Proporción } \\
\text { ponderada }\end{array}$ & $\mathbf{n}$ & $\begin{array}{l}\text { Proporción } \\
\text { ponderada }\end{array}$ & \\
\hline \multicolumn{8}{|l|}{ Total } \\
\hline & 30682 & 100 & 17216 & 100 & 13466 & 100 & \\
\hline \multicolumn{8}{|l|}{ Sexo } \\
\hline Mujer & 17216 & 51,1 & -- & -- & -- & -- & \\
\hline Hombre & 13466 & 48,9 & -- & -- & -- & -- & \\
\hline \multicolumn{8}{|l|}{ Edad } \\
\hline Media $( \pm D E)$ & $40,9( \pm 16,6)$ & -- & $40,2( \pm 16,6)$ & -- & $41,7( \pm 16,5)$ & -- & 0,041 \\
\hline \multicolumn{8}{|l|}{ Estado civil } \\
\hline Casado/conviviente & 22021 & 68,3 & 11892 & 64,7 & 10129 & 72,1 & $<0,001$ \\
\hline Otro & 8661 & 31,7 & 5324 & 35,3 & 3337 & 27,9 & \\
\hline \multicolumn{8}{|l|}{ Área de residencia } \\
\hline Urbano & 20266 & 79,7 & 11578 & 80 & 8688 & 79,4 & 0,263 \\
\hline Rural & 10416 & 20,3 & 5638 & 20 & 4778 & 20,6 & \\
\hline \multicolumn{8}{|l|}{ Nivel de educación } \\
\hline Sin educación & 1631 & 4 & 1376 & 6,5 & 255 & 1,4 & $<0,001$ \\
\hline Primaria & 7991 & 21,6 & 4691 & 24,5 & 3300 & 18,6 & \\
\hline Secundaria & 12309 & 41,1 & 6417 & 37,9 & 5892 & 44,5 & \\
\hline Superior & 8751 & 33,2 & 4732 & 31,1 & 4019 & 35,5 & \\
\hline \multicolumn{8}{|l|}{ Índice de masa corporal* } \\
\hline Bajo peso & 311 & 1 & 205 & 1,3 & 106 & 0,8 & $<0,001$ \\
\hline Normal & 11458 & 34,5 & 5851 & 31,8 & 5607 & 37,2 & \\
\hline Sobrepeso & 12225 & 40,8 & 6754 & 39,2 & 5471 & 42,4 & \\
\hline Obesidad & 6688 & 23,8 & 4406 & 27,7 & 2282 & 19,6 & \\
\hline \multicolumn{8}{|l|}{ Dominio de procedencia } \\
\hline Lima Metropolitana & 3326 & 37 & 1804 & 36,9 & 1522 & 37,2 & 0,158 \\
\hline Resto de Costa & 9142 & 25,2 & 5085 & 25,1 & 4057 & 25,3 & \\
\hline Sierra & 10874 & 25,6 & 6204 & 26,3 & 4670 & 24,9 & \\
\hline Selva & 7340 & 12,2 & 4123 & 11,8 & 3217 & 12,6 & \\
\hline \multicolumn{8}{|l|}{ Diabetes } \\
\hline No & 29822 & 96,5 & 16724 & 96,1 & 13098 & 96,8 & 0,064 \\
\hline Sí & 860 & 3,5 & 492 & 3,9 & 368 & 3,2 & \\
\hline \multicolumn{8}{|l|}{ Consumo de alcohol } \\
\hline No & 20689 & 63,9 & 13141 & 74,3 & 7548 & 53,1 & $<0,001$ \\
\hline Sí & 9993 & 36,1 & 4075 & 25,7 & 5918 & 46,9 & \\
\hline \multicolumn{8}{|l|}{ Consumo de tabaco } \\
\hline No & 27449 & 88,4 & 16657 & 95,9 & 10792 & 80,7 & $<0,001$ \\
\hline Sí & 3233 & 11,6 & 559 & 4,1 & 2674 & 19,3 & \\
\hline \multicolumn{8}{|l|}{ Quintiles de bienestar } \\
\hline Q1 (más bajo) & 9155 & 18 & 5041 & 17,8 & 4114 & 18,2 & 0,877 \\
\hline Q2 & 7906 & 20,5 & 4511 & 20,4 & 3395 & 20,6 & \\
\hline Q3 & 5899 & 21,1 & 3355 & 21,1 & 2544 & 21,1 & \\
\hline Q4 & 4542 & 20,5 & 2589 & 20,9 & 1953 & 20,2 & \\
\hline Q5 (más alto) & 3180 & 19,9 & 1720 & 19,8 & 1460 & 19,9 & \\
\hline \multicolumn{8}{|l|}{ Hipertensión arterial } \\
\hline No & 27093 & 85,5 & 15688 & 88,6 & 11405 & 82,2 & $<0,001$ \\
\hline Sí & 3589 & 14,5 & 1528 & 11,4 & 2061 & 17,8 & \\
\hline
\end{tabular}

$\mathrm{DE}=$ desviación estándar

*Se consideró como puntos de corte al bajo peso (IMC $\left.\leq 18.5 \mathrm{~kg} / \mathrm{m}^{2}\right)$, normal, sobrepeso $\left(25 \mathrm{~kg} / \mathrm{m} 2 \leq \mathrm{IMC}<30 \mathrm{~kg} / \mathrm{m}^{2}\right)$, obesidad $\left(\mathrm{IMC} \geq 30 \mathrm{~kg} / \mathrm{m}^{2}\right.$ ) Fuente: ENDES 2017 
Tabla 2. Factores asociados a hipertensión arterial en adultos peruanos según sexo (mujeres), ENDES 2017.

\begin{tabular}{|c|c|c|c|c|}
\hline \multirow{2}{*}{ Variable } & \multicolumn{4}{|c|}{ Mujeres } \\
\hline & RP (IC95\%) & Valor $p$ & $\mathrm{RPa}(\mathrm{IC95} \%)^{* *}$ & Valor $p$ \\
\hline \multicolumn{5}{|l|}{ Edad } \\
\hline & $1,05(1,05-1,06)$ & $<0,001$ & $1,05(1,05-1,06)$ & $<0,001$ \\
\hline \multicolumn{5}{|l|}{ Estado civil } \\
\hline Casado/conviviente & Ref & & Ref & \\
\hline Otro & $2,05(1,75-2,40)$ & $<0,001$ & $1,10(0,93-1,30)$ & 0,225 \\
\hline \multicolumn{5}{|l|}{ Área de residencia } \\
\hline Urbano & Ref & & Ref & \\
\hline Rural & $0,99(0,86-1,13)$ & 0,914 & - & \\
\hline \multicolumn{5}{|l|}{ Nivel de educación } \\
\hline Sin educación & Ref & & Ref & \\
\hline Primaria & $0,66(0,55-0,79)$ & $<0,001$ & $1,22(1,02-1,45)$ & 0,028 \\
\hline Secundaria & $0,30(0,24-0,37)$ & $<0,001$ & $1,08(0,84-1,40)$ & 0,528 \\
\hline Superior & $0,21(0,16-0,27)$ & $<0,001$ & $0,81(0,59-1,11)$ & 0,193 \\
\hline \multicolumn{5}{|l|}{ Índice de masa corporal* } \\
\hline Bajo peso & Ref & & Ref & \\
\hline Normal & $0,86(0,52-1,42)$ & 0,578 & $1,43(0,89-2,32)$ & 0,137 \\
\hline Sobrepeso & $1,13(0,69-1,84)$ & 0,616 & $1,86(1,15-3,01)$ & 0,011 \\
\hline Obesidad & $1,60(0,98-2,60)$ & 0,057 & $2,36(1,46-3,81)$ & $<0,001$ \\
\hline \multicolumn{5}{|l|}{ Dominio } \\
\hline Lima Metropolitana & Ref & & Ref & \\
\hline Resto de Costa & $0,75(0,62-0,91)$ & 0,004 & $0,74(0,61-0,89)$ & 0,002 \\
\hline Sierra & $0,67(0,55-0,80)$ & $<0,001$ & $0,58(0,46-0,72)$ & $<0,001$ \\
\hline Selva & $0,70(0,57-0,85)$ & 0,001 & $0,79(0,63-0,99)$ & 0,041 \\
\hline \multicolumn{5}{|l|}{ Diabetes } \\
\hline No & Ref & & Ref & \\
\hline Sí & $2,97(2,33-3,79)$ & $<0,001$ & $1,34(1,05-1,70)$ & 0,016 \\
\hline \multicolumn{5}{|l|}{ Consumo de alcohol } \\
\hline No & Ref & & Ref & \\
\hline Sí & $0,77(0,63-0,95)$ & 0,018 & $1,10(0,91-1,32)$ & 0,308 \\
\hline \multicolumn{5}{|l|}{ Consumo de tabaco } \\
\hline No & Ref & & Ref & \\
\hline Sí & $0,67(0,39-1,15)$ & 0,148 & $0,97(0,57-1,65)$ & 0,926 \\
\hline \multicolumn{5}{|l|}{ Quintiles de bienestar } \\
\hline Q1 (más bajo) & Ref & & Ref & \\
\hline Q2 & $0,76(0,63-0,92)$ & 0,005 & $0,83(0,69-0,99)$ & 0,048 \\
\hline Q3 & $0,73(0,59-0,89)$ & 0,003 & $0,67(0,54-0,85)$ & 0,001 \\
\hline Q4 & $1,05(0,86-1,30)$ & 0,587 & $0,79(0,61-1,00)$ & 0,060 \\
\hline Q5 (más alto) & $1,22(0,97-1,52)$ & 0,078 & $0,77(0,58-1,01)$ & 0,069 \\
\hline
\end{tabular}

$\mathrm{RP}=$ Razón de prevalencia cruda; $\mathrm{RPa}=$ Razón de prevalencia ajustada; $\mathrm{IC}=$ intervalo de confianza; Ref: categorías de referencia.

*Se consideró como puntos de corte al bajo peso (IMC $\leq 18.5 \mathrm{~kg} / \mathrm{m} 2)$, normal, sobrepeso $(25 \mathrm{~kg} / \mathrm{m} 2 \leq \mathrm{IMC}<30 \mathrm{~kg} / \mathrm{m} 2)$, obesidad (IMC $\geq 30 \mathrm{~kg} / \mathrm{m} 2)$

**Ajustado por todas las variables independientes que obtuvieron un valor $p<0,20$ en el análisis crudo.

Fuente: ENDES 2017 
Tabla 3. Factores asociados a hipertensión arterial en adultos peruanos según sexo (hombres), ENDES 2017.

\begin{tabular}{|c|c|c|c|c|}
\hline \multirow{2}{*}{ Variable } & \multicolumn{4}{|c|}{ Hombres } \\
\hline & RP (IC95\%) & Valor $p$ & $\mathrm{RPa}(\mathrm{IC95} \%)^{* *}$ & Valor $p$ \\
\hline \multicolumn{5}{|l|}{ Edad } \\
\hline & $1,03(1,03-1,03)$ & $<0,001$ & $1,03(1,03-1,03)$ & $<0,001$ \\
\hline \multicolumn{5}{|l|}{ Estado civil } \\
\hline Casado/conviviente & Ref & & Ref & \\
\hline Otro & $1,16(1,02-1,33)$ & 0,024 & $1,41(1,24-1,61)$ & $<0,001$ \\
\hline \multicolumn{5}{|l|}{ Área de residencia } \\
\hline Urbano & Ref & & Ref & \\
\hline Rural & $0,74(0,66-0,83)$ & $<0,001$ & $0,86(0,73-1,01)$ & 0,074 \\
\hline \multicolumn{5}{|l|}{ Nivel de educación } \\
\hline Sin educación & Ref & & Ref & \\
\hline Primaria & $0,57(0,44-0,74)$ & $<0,001$ & $0,87(0,69-1,09)$ & 0,246 \\
\hline Secundaria & $0,43(0,33-0,55)$ & $<0,001$ & $0,94(0,74-1,20)$ & 0,654 \\
\hline Superior & $0,43(0,33-0,55)$ & $<0,001$ & $0,85(0,64-1,13)$ & 0,287 \\
\hline \multicolumn{5}{|l|}{ Índice de masa corporal* } \\
\hline Bajo peso & Ref & & Ref & \\
\hline Normal & $1,08(0,54-2,12)$ & 0,822 & $1,11(0,64-1,91)$ & 0,703 \\
\hline Sobrepeso & $1,79(0,91-3,52)$ & 0,087 & $1,75(1,01-3,02)$ & 0,043 \\
\hline Obesidad & $2,87(1,46-5,63)$ & 0,002 & $2,84(1,64-4,91)$ & $<0,001$ \\
\hline \multicolumn{5}{|l|}{ Dominio } \\
\hline Lima Metropolitana & Ref & & Ref & \\
\hline Resto de Costa & $0,78(0,68-0,91)$ & 0,001 & $0,80(0,69-0,92)$ & 0,003 \\
\hline Sierra & $0,63(0,54-0,73)$ & $<0,001$ & $0,73(0,61-0,87)$ & 0,001 \\
\hline Selva & $0,67(0,56-0,80)$ & $<0,001$ & $0,77(0,64-0,93)$ & 0,008 \\
\hline \multicolumn{5}{|l|}{ Diabetes } \\
\hline No & Ref & & Ref & \\
\hline Sí & $2,10(1,67-2,63)$ & $<0,001$ & $1,21(0,96-1,52)$ & 0,101 \\
\hline \multicolumn{5}{|l|}{ Consumo de alcohol } \\
\hline No & Ref & & Ref & \\
\hline Sí & $0,94(0,83-1,07)$ & 0,369 & - & \\
\hline \multicolumn{5}{|l|}{ Consumo de tabaco } \\
\hline No & Ref & & Ref & \\
\hline Sí & $0,76(0,63-0,91)$ & 0,003 & $0,96(0,81-1,14)$ & 0,713 \\
\hline \multicolumn{5}{|l|}{ Quintiles de bienestar } \\
\hline Q1 & Ref & & Ref & \\
\hline Q2 & $0,95(0,80-1,12)$ & 0,566 & $0,84(0,70-1,01)$ & 0,067 \\
\hline Q3 & $1,06(0,89-1,26)$ & 0,458 & $0,76(0,61-0,95)$ & 0,017 \\
\hline Q4 & $1,29(1,08-1,54)$ & 0,004 & $0,79(0,62-1,00)$ & 0,057 \\
\hline Q5 & $1,47(1,23-1,74)$ & $<0,001$ & $0,83(0,63-1,09)$ & 0,195 \\
\hline
\end{tabular}

$\mathrm{RP}=$ Razón de prevalencia cruda; RPa= Razón de prevalencia ajustada; IC= intervalo de confianza; Ref: categoría de referencia

*Se consideró como puntos de corte al bajo peso (IMC $\leq 18.5 \mathrm{~kg} / \mathrm{m} 2)$, normal, sobrepeso ( $25 \mathrm{~kg} / \mathrm{m} 2 \leq \mathrm{IMC}<30 \mathrm{~kg} / \mathrm{m} 2)$, obesidad (IMC $\geq 30 \mathrm{~kg} / \mathrm{m} 2$ )

**Ajustado por todas las variables independientes que obtuvieron un valor $p<0,20$ en el análisis crudo.

Fuente: ENDES 2017

cuanto a cambios en estilos de vida como mejorar la alimentación, evitar el sedentarismo, evitar consumo de alcohol y/o tabaco los cuales son factores predisponentes a desarrollar enfermedades cardiovasculares.

En relación al dominio de residencia, un factor protector fue ser residente en el resto de la costa, sierra y selva en comparación con Lima Metropolitana. Esto es similar a estudios previos realizados en Venezuela y en América Latina donde se halló un mayor número de personas con HTA en zonas urbanas en comparación a zonas rurales. Asimismo, un estudio en población China evidenció que las áreas urbanizadas tuvieron mayor número de personas con HTA en comparación a las provincias. Esto puede explicarse por la problemática del sobrepeso y el sedentarismo, que se encuentran concentradas en zonas urbanas como Lima Metropolitana ${ }^{(21,25) .}$

Dentro de las limitaciones del estudio, se debe mencionar la probabilidad 
de no precisión de datos dado que es un estudio con análisis secundario. A esto se agrega que la ENDES no incluye información sobre algunos componentes que son considerados como factores asociados a HTA como el consumo de sal, la historia familiar de HTA, consumo calórico y niveles de actividad física ${ }^{(26)}$. Por otra parte, se debe mencionar que al ser un estudio realizado a través de la encuesta, cabe la posibilidad del sesgo de memoria de los entrevistados en variables como consumo de alcohol y tabaco además de los autorreportes en cuanto a la presencia de comorbilidades como diabetes. Sin embargo, la ENDES es una encuesta con representatividad nacional y con información actualizada sobre enfermedades crónicas y sus factores de riesgo, que ayuda a tener un mejor panorama de la HTA en el Perú.

En conclusión, se ha reportado que la HTA fue mayor en el sexo masculino en comparación al sexo femenino. Entre los factores asociados que aumentaron la probabilidad de HTA fueron la edad, sobrepeso y obesidad para hombres y mujeres, mientras que ser del resto de la costa, sierra y selva disminuyeron la probabilidad. La adopción de los planes de acción para la prevención y promoción de las enfermedades no transmisibles en nuestro país deben ser enfatizados sobre todo en los factores de riesgo descritos en el presente estudio.

\section{REFERENCIAS BIBLIOGRÁFICAS}

1. Kjeldsen SE. Hypertension and cardiovascular risk: General aspects. Pharmacol Res. 2018;129:95-99. DOI: 10.1016/j.phrs.2017.11.003

2. GBD 2017 Causes of Death Collaborators. Global, regional, and national age-sex-specific mortality for 282 causes of death in 195 countries and territories, 1980-2017: a systematic analysis for the Global Burden of Disease Study 2017. Lancet. 2018;392(10159):1736-1788. DOI: 10.1016/S01406736(18)32203-7

3. GBD 2017 Risk Factor Collaborators. Global, regional, and national comparative risk assessment of 84 behavioural, environmental and occupational, and metabolic risks or clusters of risks for 195 countries and territories, 1990-2017: a systematic analysis for the Global Burden of Disease Study 2017. Lancet. 2018;392(10159):P1923-1994. DOI: 10.1016/S0140-6736(18)32225-6
4. Worldwide trends in blood pressure from 1975 to 2015: a pooled analysis of 1479 population-based measurement studies with $19 \cdot 1$ million participants. Lancet. 2017;389(10064):P37-55. DOI: 10.1016/ S0140-6736(16)31919-5

5. Chobanian AV, Bakris GL, Black HR, Cushman WC, Green LA, Izzo JL Jr, et al. The Seventh Report of the Joint National Committee on Prevention, Detection, Evaluation, and Treatment of High Blood Pressure: the JNC 7 report. JAMA. 2003 May 21;289(19):2560-72.

6. Forouzanfar MH, Lui $\mathrm{P}$, Roth $\mathrm{GA}, \mathrm{Ng} \mathrm{M}$, Biryukov S, Marczak L, et al. Global Burden of Hypertension and Systolic Blood Pressure of at Least 110 to 115 mm Hg, 1990-2015. JAMA. 2017;317(2):165-182. DOI: 10.1001/jama.2016.19043

7. Rubinstein AL, Irazola VE, Calandrelli M, Chen CS Gutierrez L, Lanas F, et al. Prevalence, Awareness, Treatment, and Control of Hypertension in the Southern Cone of Latin America. Am J Hypertens. 2016;29(12):1343-1352. DOI: 10.1093/ajh/hpw092

8. Instituto Nacional de Estadistica e Informática Enfermedades No Transmisibles y Transmisibles, 2017 [Internet]. Lima: INEI; 2018 [citado el 3 de agosto de 2019]. Disponible en: https://www.inei. gob.pe/media/MenuRecursivo/publicaciones_digitales/Est/Lib1526/index.html

9. Agho KE, Osuagwu UL, Ezeh OK, Ghimire PR Chitekwe S, Ogbo FA. Gender differences in factors associated with prehypertension and hypertension in Nepal: A nationwide survey. PLoS One. 2018;13(9):e0203278. DOI: 10.1371/journal. pone.0203278

10. Tareque MI, Koshio A, Tiedt AD, Hasegawa T. Are the rates of hypertension and diabetes higher in people from lower socioeconomic status in Bangladesh? Results from a nationally representative survey. PLoS One. 2015;10(5):e0127954. DOI: 10.1371/journal.pone.0127954

11. Li B, Li F, Wang L, Zhang D. Fruit and Vegetables Consumption and Risk of Hypertension: A MetaAnalysis. J Clin Hypertens. 2016;18(5):468-76. DOI: $10.1111 /$ jch. 12777

12. Organización Panamericana de la Salud. HEARTS Paquete técnico para el manejo de las enfermedades cardiovasculares en la atención primaria de salud. Hábitos y estilos de vida saludables: asesoramiento para los pacientes [Internet]. Washington, D.C.: OPS; 2019 [citado el 5 de agosto de 2019]. Disponible en: https://iris.paho. org/handle/10665.2/50805

13. Instituto Nacional de Estadistica e Informática. ENDES 2017 [Internet]. Lima: INEI; 2017 [citado el 3 de setiembre de 2019]. Disponible en: http://iinei. inei.gob.pe/iinei/srienaho/Descarga/DocumentosMetodologicos/2017-5/CuestionarioSalud.pdf

14. Instituto Nacional de Estadistica e Informática. Encuesta Demográfica y Salud familiar 2017. Manual de la entrevistadora [Internet]. Lima: INEI; 2018 [citado el 17 de febrero de 2020]. Disponible en: https://proyectos.inei.gob.pe/endes/2018/ documentos_2018/MANUAL_DE_LA_ENTREVISTADORA_2018_ENERO.pdf

15. Hernández-Vásquez $A$, Chacón-Torrico $H$. Manipulación, análisis y visualización de datos de la encuesta demográfica y de salud familiar con el programa R. Rev Peru Med Exp Salud Publica. 2019;36(1):128-33. DOI: 10.17843/rpmesp.2019.361.4062.

16. Hernández-Vásquez A, Santero M. New 2017 ACC/AHA hypertension guideline: Implications for a Latin American country like Peru. Eur J Prev Cardiol. 2019;26(6):668-70. DOI: 10.1177/2047487318799489

17. Hernández-Vásquez A, Rojas-Roque $C$, Santero M, Ruiz-Maza JC, Casas-Bendezú M, Miranda JJ. ¿Qué representa cambiar el umbral diagnóstico de la hipertensión arterial? Guias ACC/AHA 2017 y su aplicación en Perú. Revista médica de Chile. 2019;147(5):545-56. DOI: 10.4067/S003498872019000500545

18. Rutstein SO, Johnson K. The DHS Wealth Index. DHS Comparative Reports No. 6 [Internet]. Estados Unidos: DHS; 2004 [citado el 4 de noviembre de 2018]. Disponible en: https://dhsprogram. com/publications/publication-cr6-comparativereports.cfm

19. Dua S, Bhuker M, Sharma P, Dhall M, Kapoor S. Body mass index relates to blood pressure among adults. N Am J Med Sci. 2014 Feb;6(2):89-95. DOI: 10.4103/1947-2714.127751

20. Gillis EE, Sullivan JC. Sex Differences in Hypertension: Recent Advances. Hypertension. 2016;68(6):1322-7. DOI: 10.1161/HYPERTENSIONAHA.116.06602

21. Neupane D, McLachlan CS, Sharma R, Gyawali B, Khanal V, Mishra SR, et al. Prevalence of hypertension in member countries of South Asian Association for Regional Cooperation (SAARC): systematic review and meta-analysis. Medicine. 2014 Sep;93(13):e74. DOI: 10.1097/ MD.0000000000000074

22. Bardach AE, Caporale JE, Alcaraz A, Augustovski F, Huayanay-Falconí L, Loza-Munarriz C, et al. Carga de enfermedad por tabaquismo e impacto potencial del incremento de precios de cigarrillos en el Perú. Rev Peru Med Exp Salud Pública. 2016;33(4):651-61. DOI: 10.17843/ rpmesp.2016.334.2548

23. Primatesta P, Falaschetti E, Gupta S, Marmot MG, Poulter NR. Association between smoking and blood pressure: evidence from the health survey for England. Hypertension. 2001;37(2):187-93. DOI: 10.1161/01.hyp.37.2.187

24. Landi F, Calvani R, Picca A, Tosato M, Martone AM, Ortolani E, et al. Body Mass Index is Strongly Associated with Hypertension: Results from the Longevity Check-Up 7+ Study. Nutrients. 2018;10(12):1976. DOI: 10.3390/nu10121976

25. Li Y, Wang L, Feng X, Zhang M, Huang Z, Deng Q, et al. Geographical variations in hypertension prevalence, awareness, treatment and control in China: findings from a nationwide and provincially representative survey. J Hypertens. 2018;36(1):178-87. DOI: 10.1097/HJH.0000000000001531

26. Khanal MK, Dhungana RR, Bhandari P, Gurung Y, Paudel KN. Prevalence, associated factors, awareness, treatment, and control of hypertension: Findings from a cross sectional study conducted as a part of a community based intervention trial in Surkhet, Mid-western region of Nepal. PLoS One. 2017;12(10):e0185806. DOI: 10.1371/journal.pone.0185806 